\title{
Upregulation of MUC1 by its novel activator 14-3-3 $\zeta$ promotes tumor invasion and indicates poor prognosis in lung adenocarcinoma
}

\author{
MIN XUE ${ }^{1}$ and WEIMIN TAO ${ }^{2}$ \\ ${ }^{1}$ Department of Respiratory Medicine, Shanghai Minhang Hospital, Fudan University, Shanghai 201199; \\ ${ }^{2}$ SICU, Tongren Hospital, Shanghai Jiao Tong University School of Medicine, Shanghai 200336, P.R. China
}

Received February 17, 2017; Accepted July 18, 2017

DOI: $10.3892 /$ or.2017.5948

\begin{abstract}
Lung adenocarcinoma (LAC) is currently the predominant histological subtype of lung cancer. Despite recent advancement in targeted therapies, the average 5-year survival rate is only $15 \%$, highlighting the need to identify previously unrecognized molecular events that propel cancer development. Herein, we showed knockdown of 14-3-3 $\zeta$ suppresses cell proliferation, migration and invasion capability of A549 and H1299 cells. MUC1 was then identified as a novel target of 14-3-3 $\zeta$ protein. Overexpression of MUC1 is found to induce epithelial-mesenchymal transition and promote metastasis of lung cancer cells, while knockdown of 14-3-3 $\zeta$ can completely abolish the oncogenic function of MUC1. Furthermore, we unraveled a novel mechanism that 14-3-3 $\zeta$ activates $\mathrm{NF}-\kappa \mathrm{B}$ signaling pathway, and therefore enhanced $\mathrm{MUC1} / \mathrm{NF}-\kappa \mathrm{B}$ feedback loop to upregulate MUC1 expression. From a clinical point of view, we evaluated the expression of 14-3-3ל and MUC1 in GSE68465 datasets, in which high expression of 14-3-3 $\zeta$ and MUC1 emerged as poor prognostic factors in LAC patients. In conclusion, we provide novel evidence that $14-3-3 \zeta$ regulates $\mathrm{MUC1}$ through $\mathrm{MUC1} / \mathrm{NF}-\kappa \mathrm{B}$ feedback loop. 14-3-3 $\zeta$ and MUC1 is a promising prognostic biomarker for lung cancer patients and therapeutic targeting of 14-3-3 $\zeta$ and MUC1 may be a potential treatment option for patients with LAC.
\end{abstract}

\section{Introduction}

Lung cancer is the most common cause of cancer related mortality worldwide (1). Lung adenocarcinoma (LAC) is currently the predominant histological subtype of lung cancer and accounts for $38 \%$ of all newly diagnosed lung cancers (2). Despite recent advancement in targeted therapies such as

Correspondence to: Dr Weimin Tao, SICU, Tongren Hospital, Shanghai Jiao Tong University School of Medicine, 1111 Xianxia Road, Shanghai 200336, P.R. China

E-mail: taoweimin2016@163.com

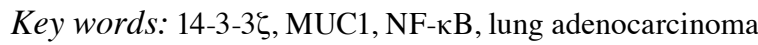

EGFR tyrosine kinase inhibitors, the average 5-year survival rate is only $15 \%$, highlighting the desperate need for additional therapeutic strategies $(3,4)$. This poor outcome is largely due to the delay of early diagnosis and lack of effective treatment against metastasis (5). Evidence suggests once they have developed metastatic disease, only $15 \%$ patients survive for 1 year after surgery with almost no long-term survivors (6). Therefore, identification of previously unrecognized molecular events that propel cancer development, particularly metastasis, would not just be of biological relevance, but also be of clinical significance allowing development of novel biomarkers or drug targets for the management of lung cancer patients.

14-3-3 $\xi$, belongs to scaffold 14-3-3 proteins family, regulates multiple signal transduction pathways and acts as an oncogene in cancer. 14-3-3 $\xi$ was initially identified as metastasis enhancer genes through comparing lung cancer cell lines with different metastatic potentials (7). Furthermore, 14-3-3 $\zeta$ was demonstrated to promote invasiveness of cancer cells by activating epithelial-mesenchymal transition (EMT) pathway (8-10). It is of note that $14-3-3 \zeta$ has been recently identified as a clinically relevant prognostic marker for breast cancer, prostate cancer, gastric cancer, and head and neck cancer (11-14). Given the important role of 14-3-3\} in carcinogenesis, particularly tumor metastasis, 14-3-3 $\zeta$ may serve as a promising biomarker or therapeutic target for lung adenocarcinoma. However, the oncogenic function and clinical relevance of $14-3-3 \zeta$ in lung adenocarcinoma remains largely unexplored.

Based upon this important gap in knowledge in the literature, we envisaged this study to interrogate the molecular contributions of $14-3-3 \xi$ in lung adenocarcinoma, identify novel downstream target of 14-3-3 $\zeta$, and decipher whether these genes may have translational relevance as clinicallyrelevant disease biomarkers or therapeutic target.

\section{Materials and methods}

Cell cultures. The cell lines A549 and H1299 were purchased from the American Type Culture Collection (ATCC). Cells were cultured in Dulbecco's modified Eagle's medium with $10 \% \mathrm{FBS}$ and $1 \%$ penicillin-streptomycin at $37^{\circ} \mathrm{C}$ in a humidified atmosphere of $5 \% \mathrm{CO}_{2}$. These cell lines were 
periodically authenticated using a panel of genetic and epigenetic biomarkers.

Plasmid, siRNA and transfection. Validated siRNA for 14-3-3ל and its negative control were purchased from Ambion. LAC cells were seeded in 6-well plate and transfected with siRNA at a final concentration of $50 \mathrm{nmol} / 1$ using Lipofectamine RNAi MAX (Invitrogen) and Opti-MEM (Gibco) according to the manufacturer's instructions when the cell density reached $30-50 \%$ confluence. A549 and H1299 cells were also stably transfected with a control pHR-CMV vector or one expressing MUC1-C according to a previous study (15). The transfection efficiency was evaluated at 48 and $72 \mathrm{~h}$ time points for evaluating corresponding changes in the mRNA and protein expression, respectively.

Cell proliferation assays. Cells with different treatment were seeded at 2000 cells per well in 96-well plates following which $20 \mu \mathrm{l}$ MTT reagent (Sigma) was added to each well with $200 \mu$ l culture medium. The MTT mixture medium were replaced by $150 \mu \mathrm{l}$ dimethyl sulfoxide after being incubated for $4 \mathrm{~h}$ at $37^{\circ} \mathrm{C}$ in a $5 \% \mathrm{CO}_{2}$ incubator. The time points were set at $0,24,48$ and $72 \mathrm{~h}$. Optical densities were determined using the Infinite 200 Pro multi-readers and i-control 1.10 software (Tecan).

Migration and invasion. Cell migration and invasion assays were performed using Boyden chambers (Corning) using $8 \mu \mathrm{m}$-size pore membrane coated with Matrigel (for invasion assays) or without Matrigel (for migration assays). The Transfected cells were seeded onto inserts at $2 \times 10^{5}$ cells in $200 \mu \mathrm{l}$ serum-free medium and transferred to lower wells with $600 \mu \mathrm{l}$ culture medium containing 10\% FBS. After $24 \mathrm{~h}$ incubation at $37^{\circ} \mathrm{C}$ in a $5 \% \mathrm{CO}_{2}$ incubator, non-invading cells were removed by scraping the top surface of the membrane. Invaded cells on the bottom of the membrane were thereafter fixed and stained by using Diff-Quick Stain kit (Thermo Scientific) according to the manufacturer's instructions. The stained cells were counted from 10 different fields randomly using a light microscope.

RNA extraction and $q P C R$. Cells were harvest at $24 \mathrm{~h}$ after transfection. Total RNA was extracted using TRIzol (Invitrogen) reagent according to the manufacturer's protocol. The qRT-PCR assays were performed using QuantStudio 6 Flex Real-time PCR System (Applied Biosystems). For geneexpression analysis, $2 \mu \mathrm{g}$ of total RNA was synthesized to cDNA by using GoScript ${ }^{\mathrm{TM}}$ Reverse Transcription System (Promega) and the Fast SYBR Green Master Mix (Applied Biosystems). The relative expression of target genes was normalized against $\beta$-actin using $2^{-\Delta \Delta c t}$ method. The sequences of all primers were shown as follows: E-cadherin: forward, AGCCCCGCCTTATGATTCTCTG, reverse, TGCCCCA TTCGTTCAAGTAGTCAT; ZEB1: forward, AGCGCTT CTCACACTCTGGGTCTT, reverse, CCTGCCTCTTCCTG CTCTGTGC; and $\beta$-actin: forward, AGAGCTACGA GCTGCCTGAC, reverse, AGCACTGTGTTGGCGTACAG. For miRNA analysis, qRT-PCR was conducted using TaqMan ${ }^{\circledR}$ MicroRNA Reverse Transcription kit and TaqMan Universal PCR Master Mix kit (Applied Biosystems) according to manufacturer's instructions. Relative expression of miR-200c was determined by $2-{ }^{\Delta \Delta c t}$ method using U6 as normalizer. Taqman primers for miR-200c and U6 were purchased from Ambion.

Immunoblot analysis. Cellular proteins were separated by SDS-PAGE gel and transferred onto a polyvinylidene difluoride(PVDF) membrane (Millipore) using Bio-Rad electrophoresis system. The PVDF membrane was blocked in $5 \%$ non-fat milk for $1 \mathrm{~h}$ at room temperature and the diluted primary antibodies (1-1000) were incubated with the target protein overnight at $4^{\circ} \mathrm{C}$. Precipitates and lysates not subjected to precipitation were analyzed by immunoblotting with the following antibodies: anti-14-3-3 (sc-1019) and anti-aPKC antibodies (sc-216) were from Santa Cruz Biotechnology; and anti-P-aPKC ${ }^{\text {Thr560 }}$ (CG1453) antibody was purchased from Cell

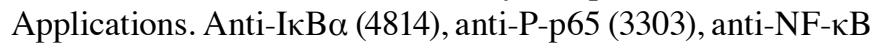
p65 (8242), and anti- $\beta$-actin (4970) antibodies were from Cell Signaling Technology. Immunoreactive complexes were detected using horseradish peroxidase-conjugated secondary antibodies (1:2000) and an enhanced chemiluminescence (ECL) detection system.

Immunoprecipitation. Cells were lysed after transfection for $48 \mathrm{~h}$ using NP-40 lysis buffer containing protease inhibitor cocktail (Thermo Scientific). Soluble proteins were immunoprecipitated with anti-MUC1, then $5 \mu \mathrm{g}$ antibody was incubated with $500 \mu \mathrm{l}$ cell lysate overnight at $4^{\circ} \mathrm{C}$. The solution was incubated with $100 \mu \mathrm{l}$ pre-cleared Protein G beads (Thermo Scientific) for $2 \mathrm{~h}$ at room temperature. The bead lysate was prepared for western blotting after being washed and boiled with 1X SDS-PAGE buffer (Beyotime Biotechnology).

Luciferase. HEK293T cells were cultured in a 96-well plate and transfected with an empty pGL3 luciferase reporter vector, a pMUC1-Luc vector, and SV-40-Renilla-Luc as an internal control in the presence of Lipofectamine 3000 reagent (Invitrogen). After $24 \mathrm{~h}$, the transfected cells were lysed using passive lysis buffer according to the instruction of Promega Dual-Luciferase reporter system kit and the lysates were analyzed using the dual luciferase assay system (Promega).

Statistical analysis. All statistical analysis was performed using the GraphPad Prism Ver. 6.0 programs (GraphPad Software Inc. San Diego, CA). All data were expressed as mean \pm SD Statistical differences between groups were determined by Wilcoxon's signed rank test, the $\chi^{2}$ test or Mann-Whitney U test. Kaplan-Meier analysis and log-rank test were used to estimate and compare survival rates of LAC patients with high and low 14-3-3 $\zeta$ or MUC1 expression. The cut-off values were established by X-tile program (X-tile software version 3.6.1, Yale University School of Medicine, New Haven, CT, USA) to calculate cut-off value (16). All P-values were 2-sided, and those $<0.05$ were considered statistically significant.

\section{Results}

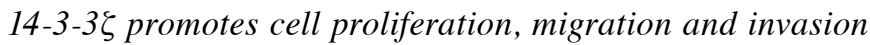
in lung adenocarcinoma. Since the role of 14-3-3६ remains largely unexplored in lung adenocarcinoma, we initially investigated whether knockdown of 14-3-35 could lead to the alteration of biological function in lung cancer cell lines. 
A

\section{A549}
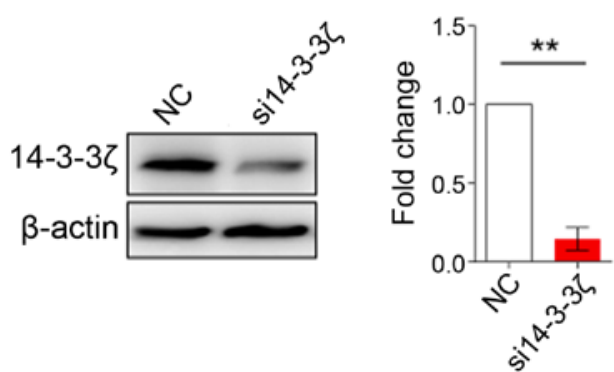

$\mathbf{B}$

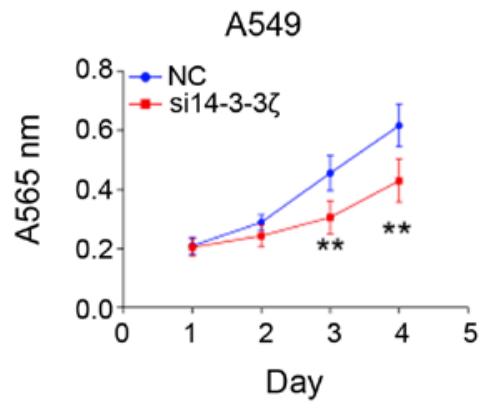

C
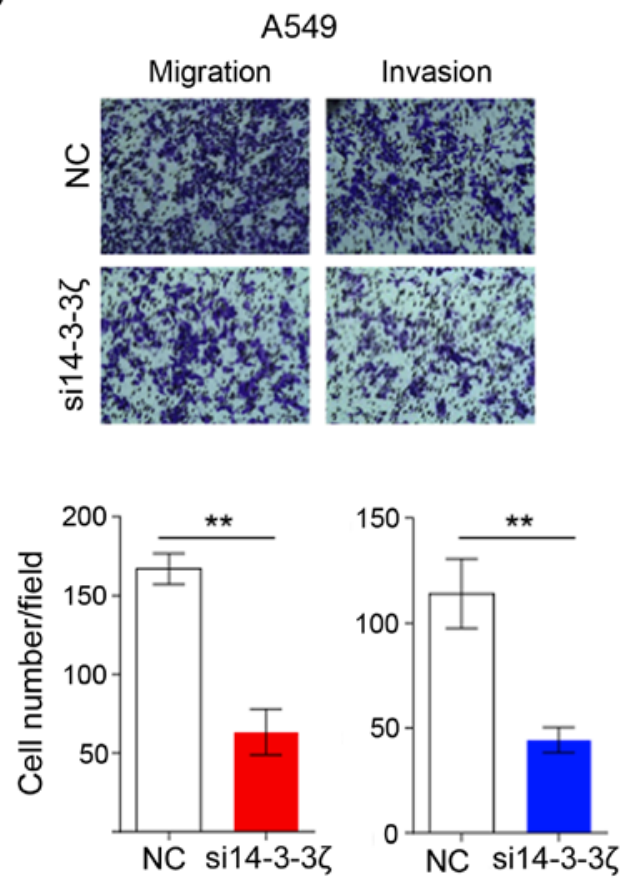

H1299
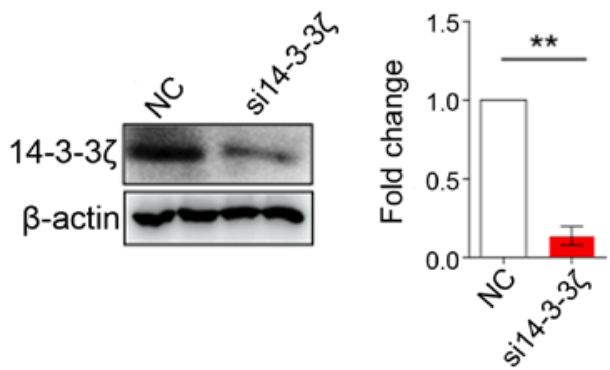

H1299
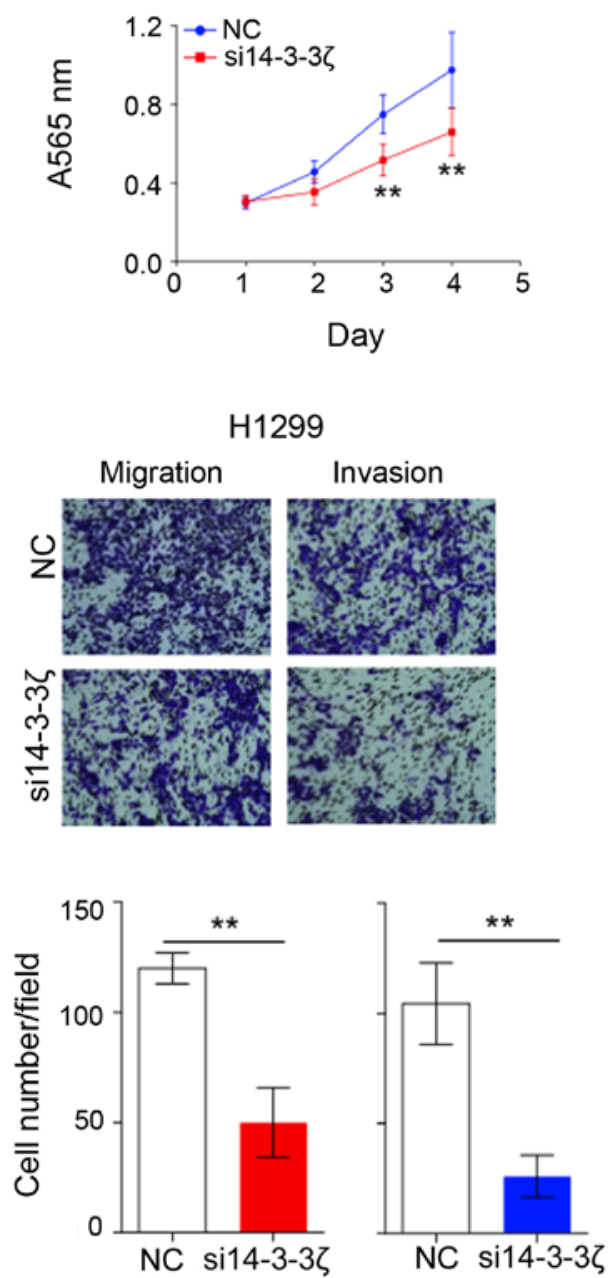

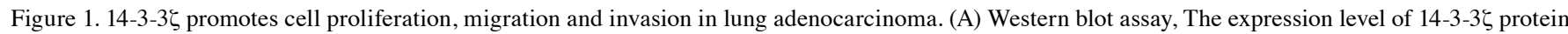

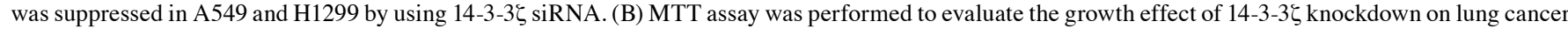
cells. (C) Transwell and Matrigel invasion assays to investigate the functional role of $14-3-3 \zeta$ on cell migration and invasion. ${ }^{* *} \mathrm{P}<0.01$.

As shown in Fig. 1A, we successfully reduced 14-3-3 $\zeta$ expression in A549 and H1299 by using RNA interference (RNAi) method according to a previously reported study (9). We subsequently performed MTT assay to evaluate the growth effect of 14-3-3 $\zeta$ knockdown on lung cancer cells. The cell growth of A549 and H1299 cells was significantly suppressed by 14-3-3 $\zeta$ siRNA treatment compared to the control group $(\mathrm{P}<0.01$, Fig. 1B), suggesting $14-3-3 \zeta$ protein plays a critical role in promoting cell proliferation in lung cancer. Furthermore, we also performed Transwell and Matrigel invasion assays to investigate the functional role of 14-3-3 $\zeta$ on cell migration and invasion. As expected, the decreased expression of 14-3-3ל resulted in an impairment of the invasive ability of both A549 and H1299 cells (Fig. 1C). Collectively, we showed interference with 14-3-3 $\zeta$ expression caused by suppression of cell growth as well as inhibition of cell migration and invasion in lung cancer cells.

MUC1 is identified as a novel target of 14-3-3\} in lung adenocarcinoma. Owing to its oncogenic role in lung cancer, 14-3-3 $\zeta$ may affect certain signaling pathways and promote the development of lung cancer. Several important surface 
A

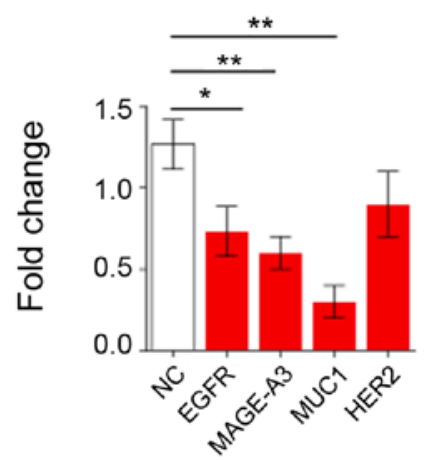

B

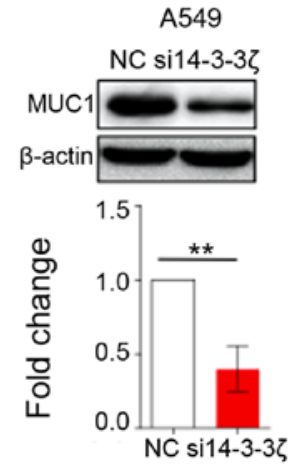

H1299

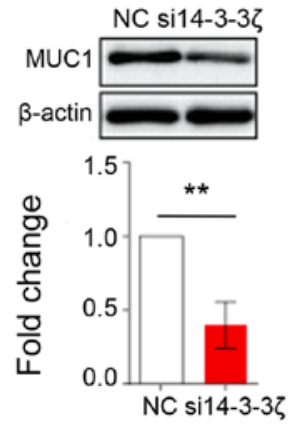

C

A549

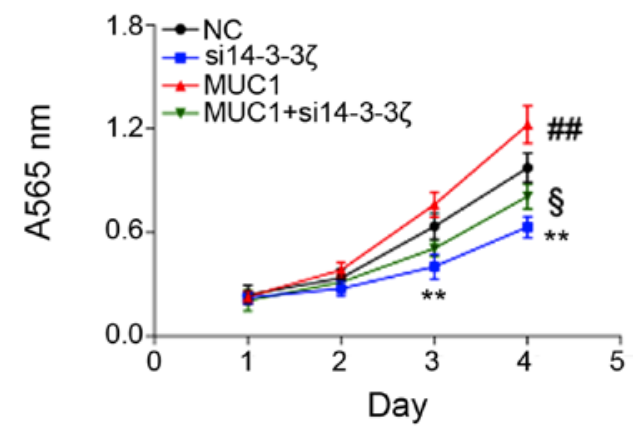

D
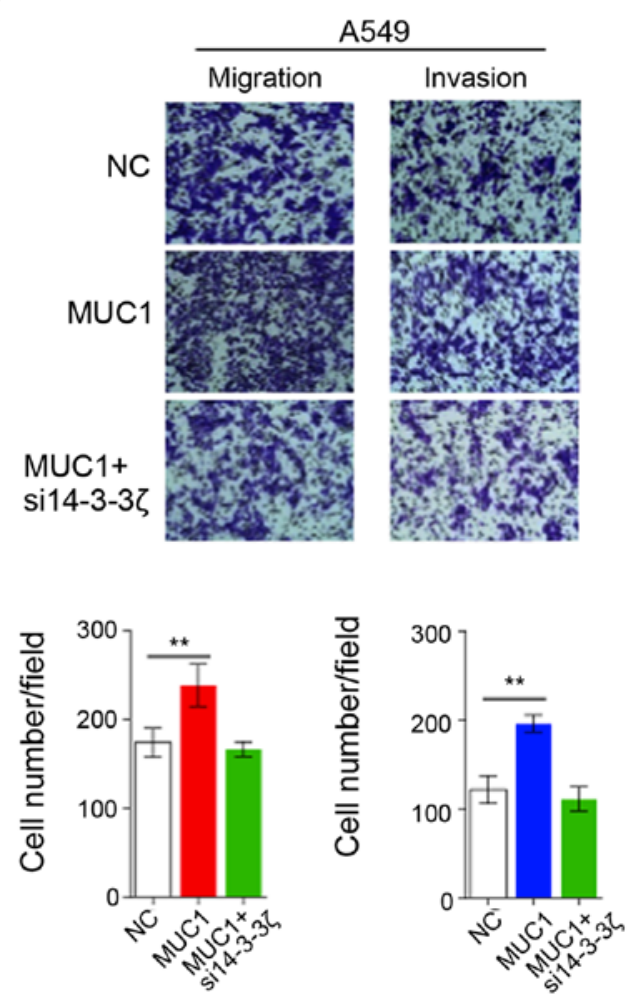
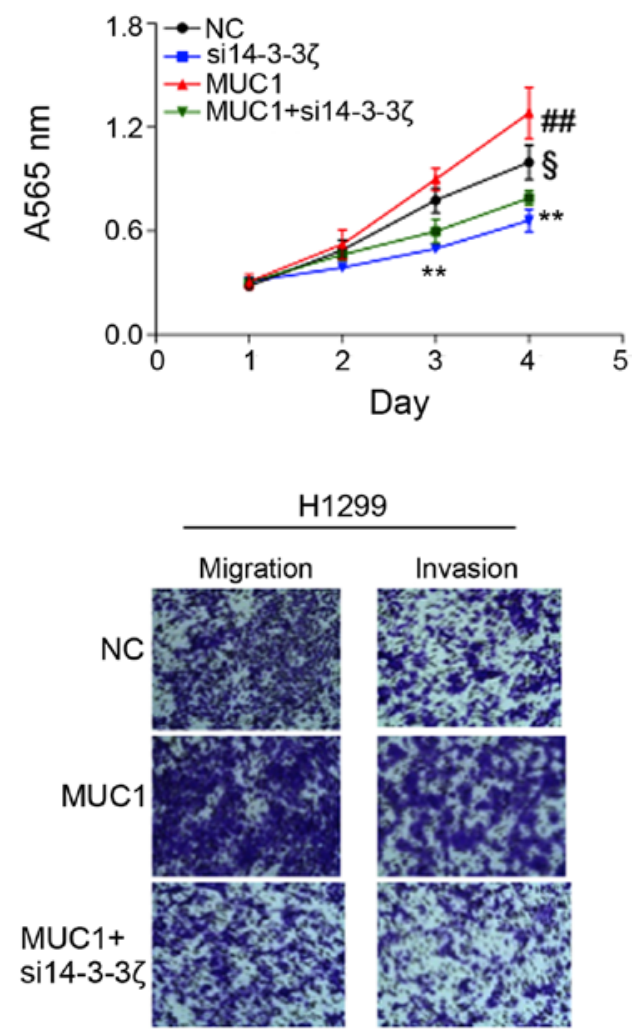

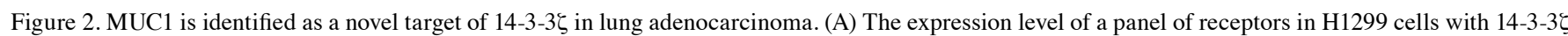
siRNA or negative control treatment. ${ }^{*} \mathrm{P}<0.05,{ }^{* * *} \mathrm{P}<0.01$. (B) MUC1 protein level in A549 and H1299 cells with 14-3-3६ siRNA or negative control treatment. (C) MTT assay was performed to evaluate the growth effect of MUC1 overexpression alone, the combination of MUC1 overexpression and 14-3-3 $\zeta$ knock-

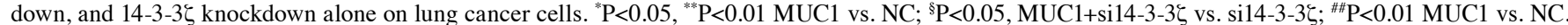
(D) Transwell and Matrigel invasion assays to investigate the functional role of MUC1 overexpression alone, the combination of MUC1 overexpression and 14-3-3६ knockdown, and 14-3-3६ knockdown alone on cell migration and invasion. ${ }^{*} \mathrm{P}<0.05,{ }^{* *} \mathrm{P}<0.01$.

receptors have been found upregulated in lung adenocarcinoma for sustaining proliferative or metastatic signaling. We hypothesized that 14-3-3६ may have impact on the expression of such surface receptors. Based on our assumption, we first 
evaluated the expression level of a panel of receptors (EGFR, MAGE-A3, MUC1 and HER2), which were reported previously as activators of lung cancer, in 14-3-3 $\zeta$ siRNA treated and untreated H1299 cells. The results showed the expression of EGFR, MAGE-A3 and MUC1 was significantly reduced, while HER 2 remains unchanged upon $14-3-3 \zeta$ siRNA treatment (Fig. 2A). Notably, the expression of MUC1 was severely decreased by $70 \%$ compared to EGFR (30\%) and MAGE-A3 (40\%). To confirm that the expression of MUC1 can be regulated by 14-3-35, we also treated A549 and H1299 cells with 14-3-3६ siRNA and then measured protein level of MUC1 by western blotting. Consistently, MUC1 protein level is markedly downregulated when $14-3-3 \xi$ expression is decreased in A549 and H1299 cells (Fig. 2B).

Since 14-3-3 $\zeta$ is able to affect expression of MUC1, we hypothesized that 14-3-3 $\zeta$ may also have impact on biological function of MUC1. As depicted in Fig. 2C, overexpression of MUC1 significantly enhanced proliferation of both A549 and H1299 cells compared to control cells; however, 14-3-3\} knockdown effectively inhibits MUC1 function and results in the suppression of cell growth. Similar to MTT assay, MUC1 overexpression promotes cell migration and invasion, while knockdown of 14-3-3 $\zeta$ completely neutralized function of MUC1 (Fig. 2D). Our data clearly showed MUC1 is a novel target of $14-3-3 \xi$ in lung cancer. $14-3-3 \zeta$ plays a crucial role for MUC1-mediated phenotypic characteristics of lung cancer cells.

14-3-3६ upregulates MUC1 expression through enhancing $M U C 1 / N F \kappa B$ feedback loop. Recently, MUC1 COOHterminal subunit (MUC1-C) cytoplasmic domain was reported to bind directly to $\mathrm{p} 65$, trigger $\mathrm{p} 65$ release from its inhibitor I $\mathrm{K} \mathrm{B} \alpha$, leading to the constitutive activation of the NF- $\kappa B$ pathway. Furthermore, the MUC1-C/p65 complex can bind to NF- $\mathrm{KB}$ target genes, including the promoter of the MUC1 gene itself, thus resulting in auto-inductive regulatory loop (17). Noteworthy, 14-3-3 $\zeta$ was demonstrated to activate $\mathrm{NF}-\kappa \mathrm{B}$ signal transduction pathway through atypical protein kinase $\mathrm{C}$ (aPKC) activation $(18,19)$, suggesting $14-3-3 \zeta$ may affect MUC1 expression through MUC1/NF- $\mathrm{KB}$ feedback loop. We therefore first confirmed that the activity of aPKC is inhibited by the knockdown of 14-3-3 5 in H1299 and A549 cells, as measured by the phosphorylation of Thr-560. We further observed that 14-3-3 $\zeta$ knockdown caused increased level of IкB $\alpha$ subunit and decreased phosphorylation of $\mathrm{p} 65$, implicating that $14-3-3 \xi$ knockdown inhibited aPKC activity and its target NF- $\kappa \mathrm{B}$ signaling pathway (Fig. 3A).

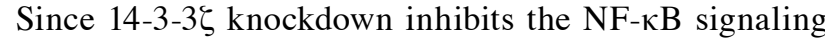
pathway, we assume 14-3-3 may affect the formation of MUC1-C/p65 complex. As expected, knockdown of 14-3-3\} attenuates binding of MUC1-C and p65 (Fig. 3B). Previous study showed MUC1-C/p65 complex is able to bind to the promoter region (NF- $\kappa \mathrm{B}$ responsive element) of MUC1 (Fig. 3C) and induce its mRNA expression (17). We thereafter performed ChIP assay with MUC1-C antibody to determine whether occupancy of MUC1 promoter by MUC1-C was decreased by silencing 14-3-3६. Consistent with our hypothesis, the binding of MUC1 to the NF- $\mathrm{kB}$ responsive element was significantly decreased when silencing $14-3-3 \zeta$ in H1299 and A549 cells (Fig. 3C). To determine whether 14-3-3 $\zeta$ affects
Table I. The correlation between clinicopathological characteristics, $\mathrm{MUC1}$ and 14-3-3६ expression in lung adenocarcinomas.

\begin{tabular}{|c|c|c|c|c|c|c|}
\hline \multirow[b]{2}{*}{ Characteristics } & \multicolumn{2}{|c|}{ MUC1 } & \multirow[b]{2}{*}{ P-value } & \multicolumn{2}{|c|}{$14-3-3 \xi$} & \multirow[b]{2}{*}{ P-value } \\
\hline & Low & High & & Low & High & \\
\hline \multicolumn{7}{|l|}{ Gender } \\
\hline Female & 160 & 57 & 0.305 & 169 & 48 & 0.1863 \\
\hline Male & 169 & 52 & & 159 & 62 & \\
\hline \multicolumn{7}{|l|}{ Age } \\
\hline$\geq 65$ & 156 & 56 & 0.368 & 168 & 44 & 0.0539 \\
\hline$<65$ & 173 & 53 & & 160 & 66 & \\
\hline \multicolumn{7}{|l|}{ Smoking } \\
\hline Never & 31 & 17 & 0.0585 & 35 & 13 & 0.8187 \\
\hline Past & 202 & 63 & & 204 & 61 & \\
\hline Current & 28 & 4 & & 24 & 8 & \\
\hline Unknown & 68 & 25 & & 65 & 28 & \\
\hline \multicolumn{7}{|l|}{ Grade } \\
\hline Well & 45 & 15 & 0.8522 & 50 & 10 & 0.3415 \\
\hline Moderate & 155 & 53 & & 156 & 52 & \\
\hline Poor & 126 & 39 & & 118 & 47 & \\
\hline Unknown & 3 & 2 & & 4 & 1 & \\
\hline \multicolumn{7}{|c|}{ Lymph node metastasis } \\
\hline Negative & 232 & 66 & 0.0695 & 222 & 76 & 0.8761 \\
\hline Positive & 97 & 43 & & 106 & 34 & \\
\hline \multicolumn{7}{|l|}{ Stage } \\
\hline I & 212 & 63 & 0.4419 & 209 & 66 & 0.6536 \\
\hline II & 69 & 26 & & 71 & 24 & \\
\hline III & 48 & 20 & & 48 & 20 & \\
\hline
\end{tabular}

Several pathological variables showed tendency to correlate with the expression level of MUC1 or 14-3-3\}. The P-value is shown as bold text.

activation of MUC1 transcription, we first overexpressed MUC1 in H1299 and A549 cells. In line with previous studies, MUC1 overexpression can successfully promote the activity of MUC1-promoter luciferase reporter. However, such positive effect was significantly attenuated by 14-3-3 $\zeta$ knockdown (Fig. 3D), suggesting 14-3-3 $\zeta$ regulates MUC1 expression in a promoter-dependent manner.

14-3-3 $\zeta$ and MUC1 was reported to drive epithelialmesenchymal transition (EMT) in different cancer types (8,20-22). We observed that overexpression of MUC1 in H1299 and A549 cells is able to upregulate mesenchymal transcription factor ZEB1, while downregulated epithelial markers E-cadherin and inducer of epithelial differentiation miR-200c compared to the control group. In contrast, reduction of 14-3-3 $\xi$ expression upregulated ZEB1, while decreased expression of E-cadherin and miR-200c, suggesting both 14-3-3 $\zeta$ and MUC1 contribute to EMT in lung cancer (Fig. 4A and B). Furthermore, we noted that downregulation of 14-3-3 $\zeta$ suppressed function of MUC1 to induce EMT, suggesting the oncogenic function of MUC1 requires the expression of 14-3-3ל (Fig. 4C). 
A

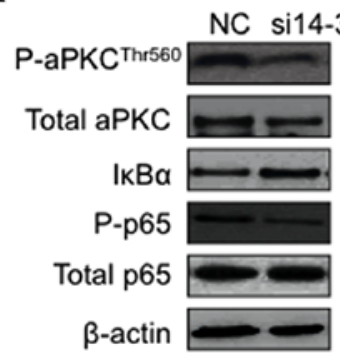

H1299
$4-3-3 \zeta$

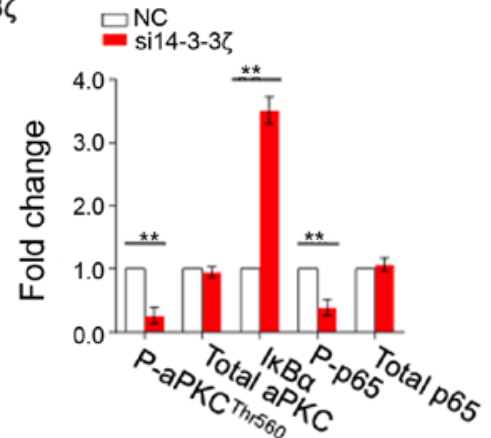

C

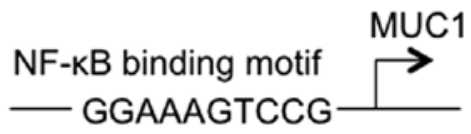

H1299

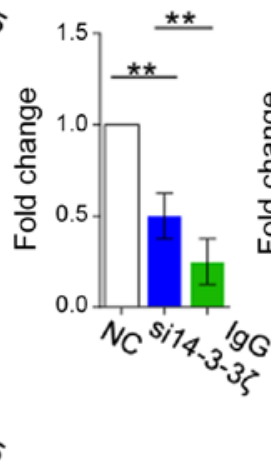

A549

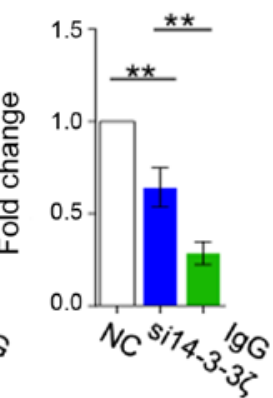

B

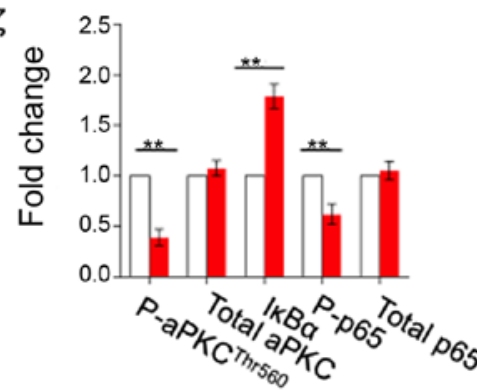

A549

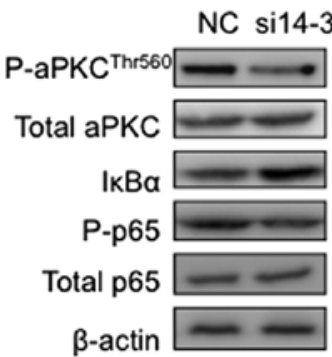

H1299
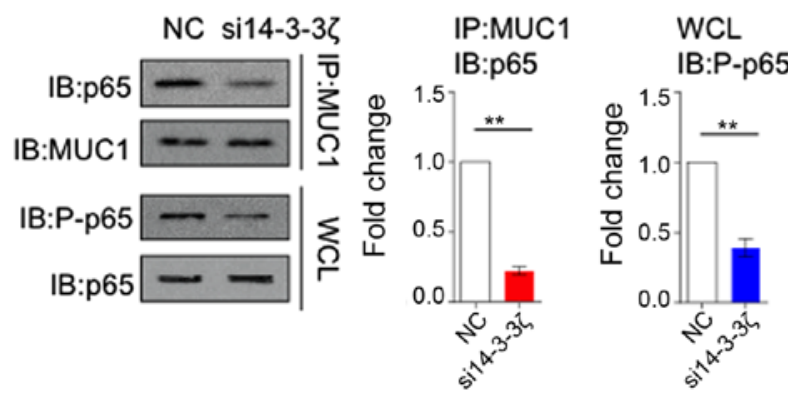

A549

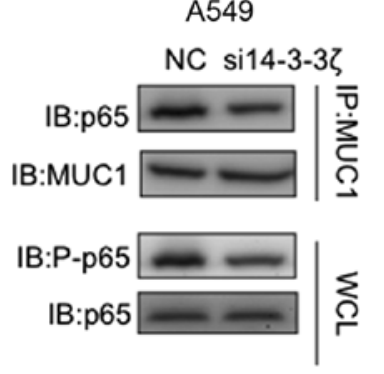

IP:MUC1 IB:p65
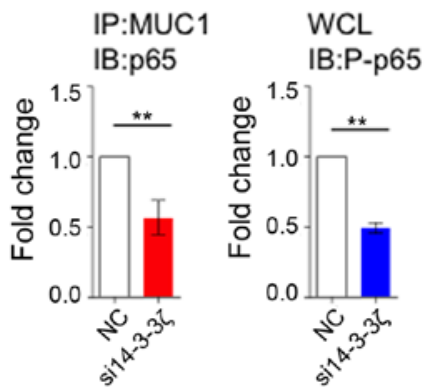

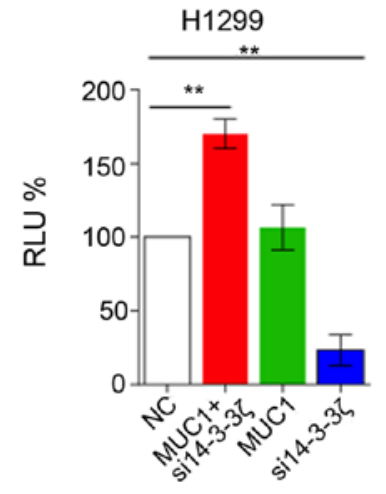

A549

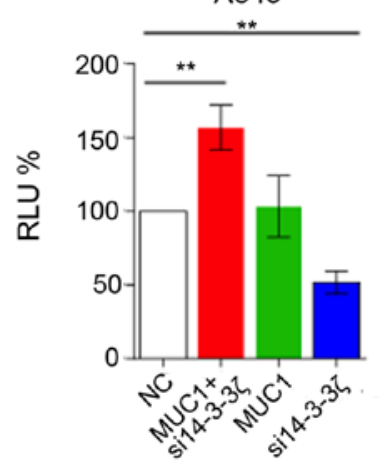

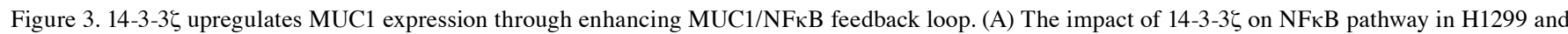

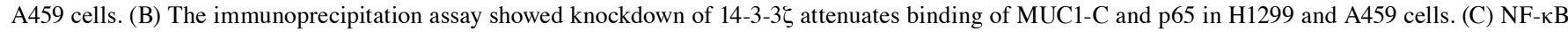
responsive element in the promoter of MUC1. ChIP assay was performed to determine whether occupancy of MUC1 promoter by MUC1-C was decreased by

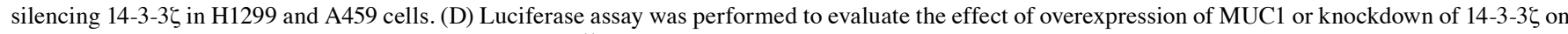
the promoter activity of MUC1 in $\mathrm{H} 1299$ and A459 cells. ${ }^{* *} \mathrm{P}<0.01$.

High expression of 14-3-35 and MUC1 is associated with poor survival in lung adenocarcinoma patients. To explore the clinical relevance of 14-3-3 $\zeta$ and MUC1 in lung cancer, we used public available gene expression omnibus (GEO) to analyze their expression levels in association with clinicopathological characteristics and survival. GSE68465 datasets were used since it was a large and multi-site lung adenocarcinoma cohort with complete follow-up information (23). The correlation between 14-3-3ל and MUC1 expression and clinicopathological characteristics is shown in Table I. Kaplan-Meier survival analysis showed the group with high expression level of $14-3-3 \xi$ and MUC1 showed remarkably poor prognosis 
A
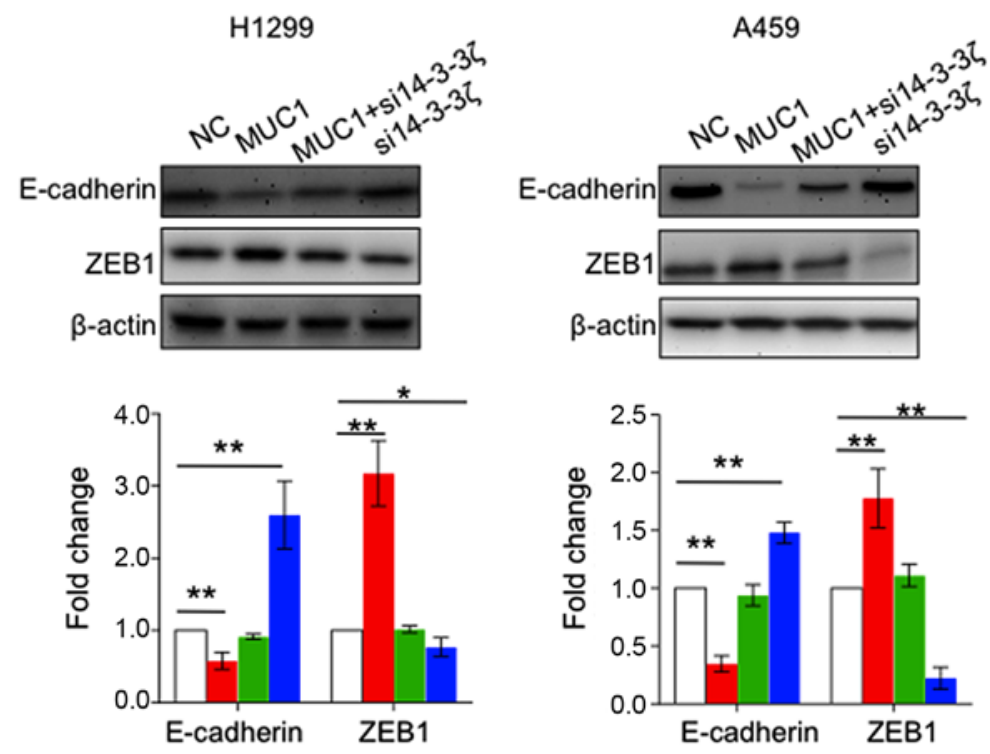

B

H1299

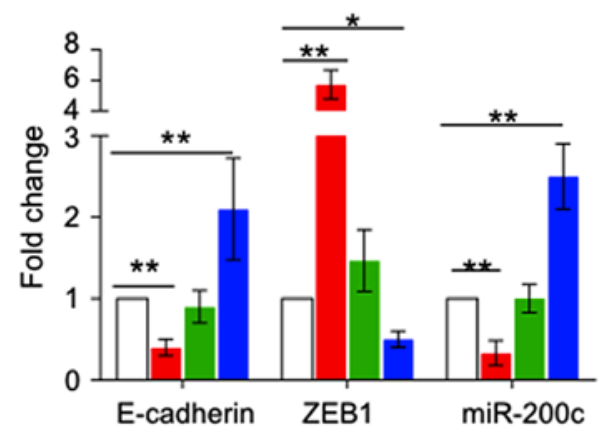

A459

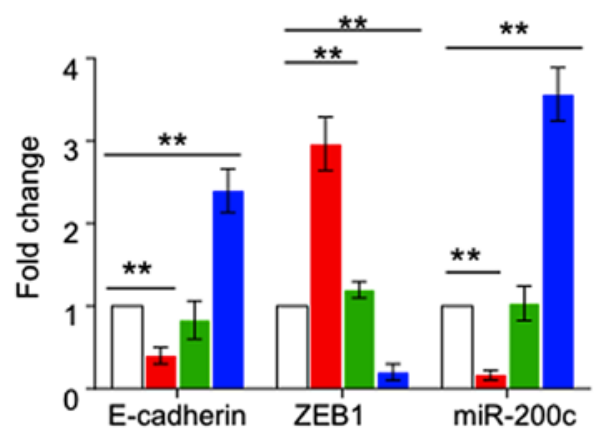

C

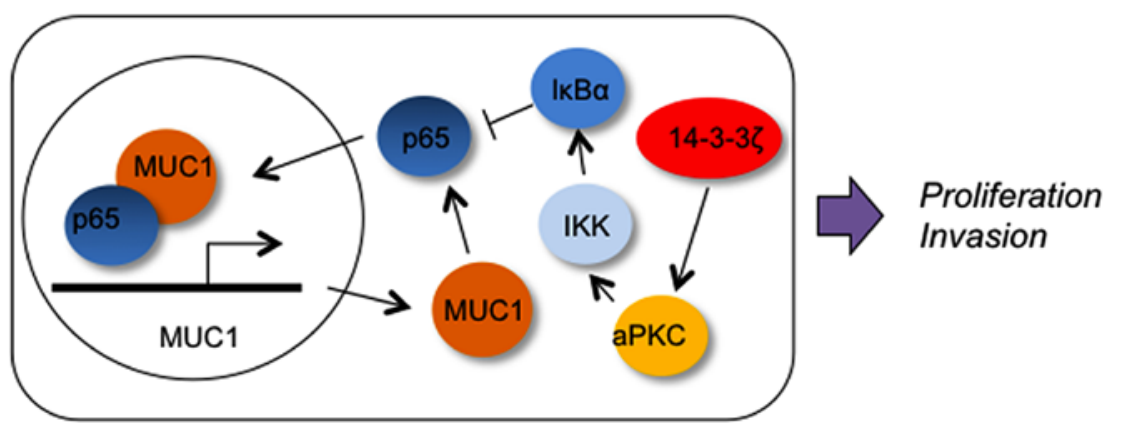

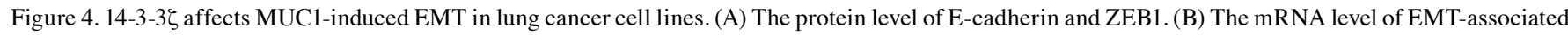

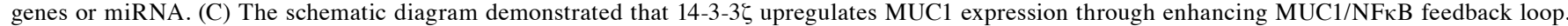
${ }^{*} \mathrm{P}<0.05 ;{ }^{* * *} \mathrm{P}<0.01$.

compared to the low expression group $(\mathrm{P}=0.0062, \mathrm{P}=0.0005$, respectively). Importantly, when we segregated patients into 4 groups based on 14-3-3 $\zeta$ levels and MUC1 expression, we discovered that the group with both high 14-3-3 $\xi$ and high MUC1 expression showed worst prognosis compared to other 3 groups (Fig. 5A).

It was reported that a subset of patients with stage I disease have poorer prognosis and may benefit significantly from adjuvant chemotherapy $(24,25)$. When we analyzed

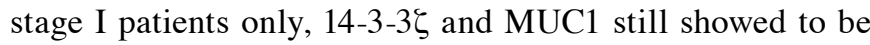
a highly predictive prognostic indicator, wherein patients in the group with high 14-3-3 $\zeta$ or MUC1 expression were more likely to have a poor outcome vs. that with low expression $(\mathrm{P}=0.0002, \mathrm{P}=0.0002$ respectively). Consistently, when we segregated patients into 4 groups based on 14-3-3 $\zeta$ levels and MUC1 expression in stage I patients, we also observed that 
A

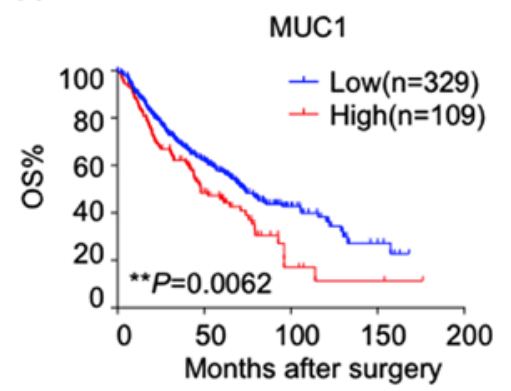

$14-3-3 \zeta$

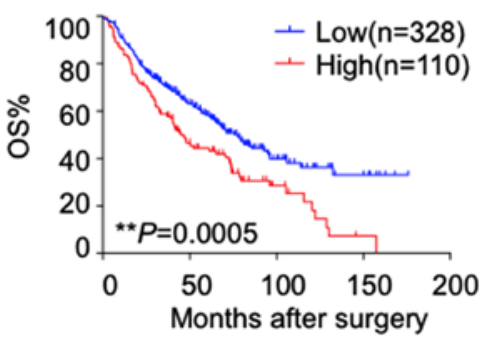

MUC1+14-3-3ל

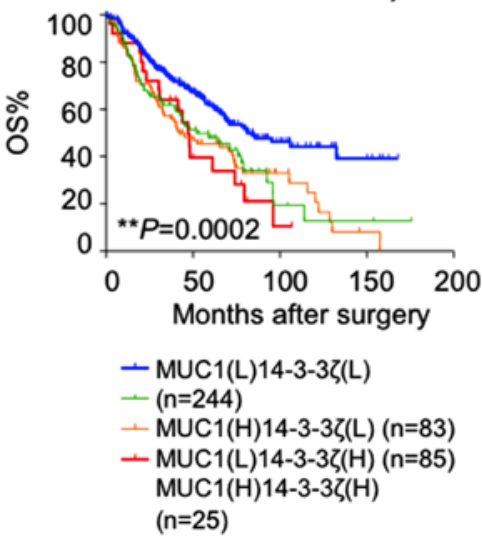

B
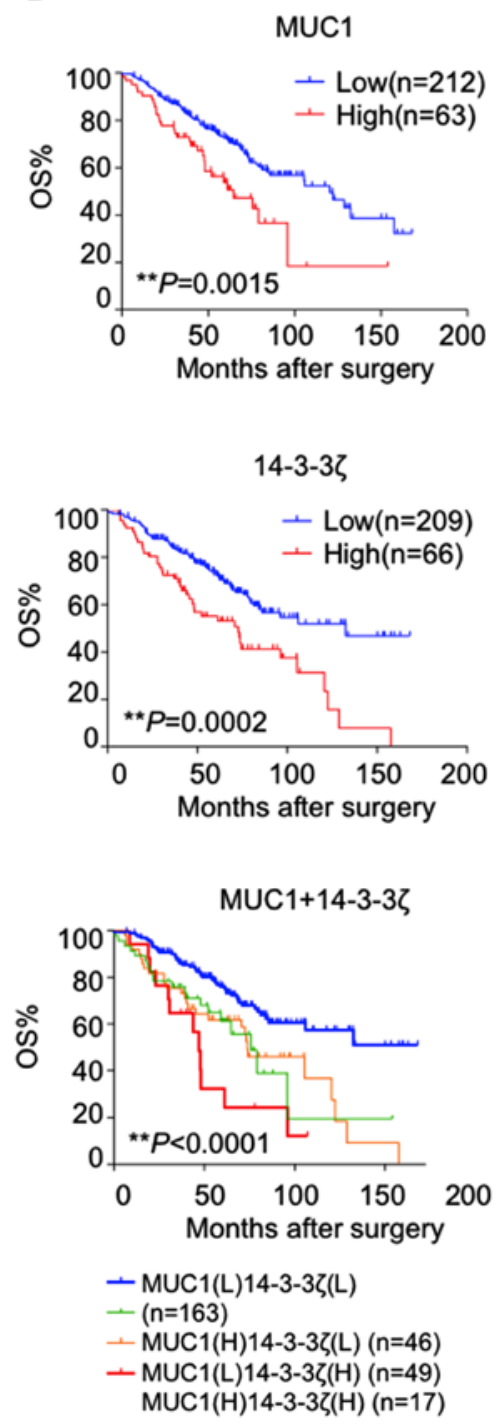

Figure 5. High expression of 14-3-3 $\zeta$ and MUC1 was associated with poor survival in lung adenocarcinoma patients. (A) Kaplan-Meier survival analysis showed the group with high expression level of 14-3-35 and MUC1 showed remarkably poor prognosis compared to the low expression group when we

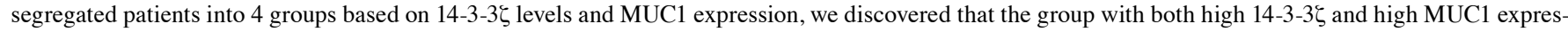
sion showed worst prognosis compared to the other 3 groups. (B) When stage I patients were analyzed only, 14-3-3६ and MUC1 still showed to be a highly predictive prognostic indicator, wherein patients in the high expression group were more likely to have a poor outcome vs. those with low-risk. Consistently, when we segregated patients into 4 groups based on 14-3-3 $\zeta$ levels and MUC1 expression in stage I patients, the group with both high 14-3-3 $\zeta$ and high MUC1 expression group showed worst prognosis compared to the other 3 groups. ${ }^{* *} \mathrm{P}<0.01$.

the group with both high 14-3-3 $\zeta$ and high MUC1 expression group showed worst prognosis compared to the other 3 groups (Fig. 5B). Collectively, these results indicate that 14-3-36 and MUC1 could successfully segregate high vs. low-risk patients with stage I disease.

\section{Discussion}

In this study, we for the first time showed Mucin 1 (MUC1) is a novel downstream target of 14-3-3 $\zeta$ in lung adenocarcinoma. Second, we unraveled a novel mechanism that 14-3-3 $\zeta$ affects MUC1 expression through MUC1/NF- $\kappa$ B feedback loop. Third, from a biological perspective, we demonstrated that both 14-3-3 $\zeta$ and MUC1 are involved in activation of EMT pathway and promoted aggressiveness of lung cancer cells, highlighting the therapeutic importance of 14-3-3 $\zeta$ and MUC1 in lung adenocarcinoma. Fourth, high expression of 14-3-3 $\zeta$ and MUC1 correlated with poor patient outcomes, highlighting its applicability as a promising prognostic biomarker in lung adenocarcinoma.

Recently, 14-3-3 $\zeta$ protein has aroused considerable interest since it was overexpressed in different types of cancer and reported to activate EMT-associated genes to promote metastasis. Our data clearly showed interference with 14-3-3 $\zeta$ expression in lung cancer cell lines caused suppression of cell growth, migration and invasion, suggesting its oncogenic role in the development of lung cancer.

To further investigate the molecular event mediated by 14-3-3 $\zeta$, we screened several important cell surface receptors which may be affected by $14-3-3 \zeta$. Of note, we observed that 
the expression of MUC1 was dramatically downregulated by silencing 14-3-3 $\zeta$ in lung cancer cells, suggesting MUC1 is a potential target of $14-3-3 \zeta$. MUC1 is a trans-membrane heterodimeric protein that is aberrantly expressed in nonsmall cell lung cancer. It was reported that over $80 \%$ of lung adenocarcinoma express high level of MUC1 (26). Of importance, MUC1 undergoes autocleavage into two subunits. The MUC1 N-terminal subunit (MUC1-N) contains glycosylated tandem repeats, while C-terminal subunit (MUC1-C) can interact with receptor tyrosine kinases, such as EGFR (27). Moreover, MUC1-C is able to bind directly to NF- $\kappa \mathrm{B}$ p65 and promotes $\mathrm{NF}-\kappa \mathrm{B}-$ mediated gene transcription including MUC1 gene itself. Our study revealed a new mechanism that 14-3-3 $\zeta$ activated NF- $\mathrm{NB}$ signaling pathway through aPKC, the hyperactivated p65 can bind more MUC1-C, and MUC1-C/ p65 complex therefore persistently activates the promoter of $\mathrm{MUC1}$, resulting in a hyperactive $\mathrm{MUC1/NF- \kappa B}$ feedback loop.

Not only MUC1 gene per se, but other NF- $\kappa \mathrm{B}$ target genes, ZEB1, for instance, could be affected by 14-3-3 $\zeta$. The available evidence showed that MUC1-C occupies the ZEB1 promoter with $\mathrm{NF}-\kappa \mathrm{B}$ and thereby promotes ZEB1 transcription. Furthermore, MUC1-C associates with ZEB1 and the MUC1-C/ZEB1 complex suppresses transcription of miR-200c, an inducer of epithelial differentiation (20). Herein, our data successfully validated that overexpression of MUC1 leads to upregulation of ZEB1, while suppressed the expression of miR-200c. More importantly, we found 14-3-3 $\zeta$ knockdown could abolish the impact of MUC1 overexpression on ZEB1 and miR-200c, implicating that the function of MUC1 to induce EMT is dependent on 14-3-3 $\zeta$ protein and 14-3-3 $\zeta$ promotes metastasis, at least in part, through $\mathrm{MUC} 1 / \mathrm{NF}-\kappa \mathrm{B}$ feedback loop.

To better appreciate the oncogenic role of $14-3-3 \xi$ and MUC1 for its contribution to lung carcinogenesis, we also investigated their correlation in association with clinical outcome. The GSE68465 datasets showed that the group with high expression level of 14-3-3 $\zeta$ and/or MUC1 showed remarkably poor prognosis compared to the low expression group. Furthermore, when we analyzed stage I patients only, 14-3-3\} and/or MUC1 still showed to be a highly predictive prognostic indicator, wherein patients in the high expression group were more likely to have a poor outcome vs. those with low-risk, suggesting that 14-3-3 $\zeta$ and MUC1 could successfully segregate high vs. low-risk patients with stage I disease.

In summary, ours is the first study to systematically interrogate the functional and clinical significance of $14-3-3 \xi$ and its novel target MUC1 in lung adenocarcinoma, and we provide comprehensive evidence that 14-3-3 $\zeta$ can regulate MUC1 through MUC1/NF- $\mathrm{B}$ feedback loop. We conclude that 14-3-3 $\zeta$ and/or MUC1 is a promising prognostic biomarker for lung cancer patients; therapeutic targeting of 14-3-3 $\zeta$ and/or MUC1 may be a potential treatment option for patients with lung adenocarcinoma.

\section{References}

1. Jemal A, Siegel R, Ward E, Hao Y, Xu J, Murray T and Thun MJ: Cancer statistics, 2008. CA Cancer J Clin 58: 71-96, 2008.

2. Travis WD: Pathology of lung cancer. Clin Chest Med 32: 669-692, 2011
3. Yang $X$, Yang $K$ and Kuang $K$ : The efficacy and safety of EGFR inhibitor monotherapy in non-small cell lung cancer: A systematic review. Curr Oncol Rep 16: 390, 2014.

4. Rossi A, Maione P, Sacco PC, Sgambato A, Casaluce F, Ferrara ML, Palazzolo G, Ciardiello F and Gridelli C: ALK inhibitors and advanced non-small cell lung cancer (Review). Int J Oncol 45: 499-508, 2014.

5. Inamura K: Diagnostic and therapeutic potential of microRNAs in lung cancer. Cancers (Basel) 9: 9, 2017.

6. Groome PA, Bolejack V, Crowley JJ, Kennedy C, Krasnik M, Sobin LH and Goldstraw P; IASLC International Staging Committee; Cancer Research and Biostatistics; Observers to the Committee; Participating Institutions: The IASLC Lung Cancer Staging Project: Validation of the proposals for revision of the $\mathrm{T}, \mathrm{N}$, and $\mathrm{M}$ descriptors and consequent stage groupings in the forthcoming (seventh) edition of the TNM classification of malignant tumours. J Thorac Oncol 2: 694-705, 2007.

7. Chen JJ, Peck K, Hong TM, Yang SC, Sher YP, Shih JY, Wu R, Cheng JL, Roffler SR, Wu CW, et al: Global analysis of gene expression in invasion by a lung cancer model. Cancer Res 61: 5223-5230, 2001.

8. Yang Y, Liu Y, He JC, Wang JM, Schemmer P, Ma CQ, Qian YW, Yao W, Zhang J, Qi WP, et al: 14-3-3 $\zeta$ and aPKC-ı synergistically facilitate epithelial-mesenchymal transition of cholangiocarcinoma via GSK-3 $\beta /$ Snail signaling pathway. Oncotarget 7: 55191-55210, 2016.

9. Huang XY, Ke AW, Shi GM, Zhang X, Zhang C, Shi YH, Wang XY, Ding ZB, Xiao YS, Yan J, et al: $\alpha \mathrm{B}$-crystallin complexes with 14-3-3 6 to induce epithelial-mesenchymal transition and resistance to sorafenib in hepatocellular carcinoma. Hepatology 57: 2235-2247, 2013.

10. Tang Y, Liu S, Li N, Guo W, Shi J, Yu H, Zhang L, Wang K, Liu S and Cheng S: 14-3-3 $\zeta$ promotes hepatocellular carcinoma venous metastasis by modulating hypoxia-inducible factor- $1 \alpha$. Oncotarget 7: 15854-15867, 2016.

11. Rüenauver K, Menon R, Svensson MA, Carlsson J, Vogel W, Andrén O, Nowak $M$ and Perner S: Prognostic significance of YWHAZ expression in localized prostate cancer. Prostate Cancer Prostatic Dis 17: 310-314, 2014.

12. Nishimura Y, Komatsu S, Ichikawa D, Nagata H, Hirajima S, Takeshita H, Kawaguchi T, Arita T, Konishi H, Kashimoto K, et al: Overexpression of YWHAZ relates to tumor cell proliferation and malignant outcome of gastric carcinoma. $\mathrm{Br} \mathrm{J}$ Cancer 108: 1324-1331, 2013.

13. Matta A, DeSouza LV, Shukla NK, Gupta SD, Ralhan R and Siu KW: Prognostic significance of head-and-neck cancer biomarkers previously discovered and identified using iTRAQlabeling and multidimensional liquid chromatography-tandem mass spectrometry. J Proteome Res 7: 2078-2087, 2008.

14. Neal CL, Yao J, Yang W, Zhou X, Nguyen NT, Lu J, Danes CG, Guo H, Lan KH, Ensor J, et al: 14-3-3zeta overexpression defines high risk for breast cancer recurrence and promotes cancer cell survival. Cancer Res 69: 3425-3432, 2009.

15. Rajabi H, Ahmad R, Jin C, Joshi MD, Guha M, Alam M, Kharbanda $S$ and Kufe D: MUC1-C oncoprotein confers androgen-independent growth of human prostate cancer cells. Prostate 72: 1659-1668, 2012.

16. Camp RL, Dolled-Filhart M and Rimm DL: X-tile: a new bioinformatics tool for biomarker assessment and outcome-based cut-point optimization. Clin Cancer Res 10: 7252-7259, 2004

17. Ahmad R, Raina D, Joshi MD, Kawano T, Ren J, Kharbanda S and Kufe D: MUC1-C oncoprotein functions as a direct activator of the nuclear factor-kappaB p65 transcription factor. Cancer Res 69: 7013-7021, 2009.

18. Tang Y, Lv P, Sun Z, Han L, Luo B and Zhou W: 14-3-3 up-regulates hypoxia-inducible factor- $1 \alpha$ in hepatocellular carcinoma via activation of PI3K/Akt/NF- $\kappa$ B signal transduction pathway. Int J Clin Exp Pathol 8: 15845-15853, 2015.

19. Tong S, Xia T, Fan K, Jiang K, Zhai W, Li JS, Wang SH and Wang JJ: $14-3-3 \zeta$ promotes lung cancer cell invasion by increasing the Snail protein expression through atypical protein kinase $\mathrm{C}(\mathrm{aPKC}) / \mathrm{NF}-\kappa \mathrm{B}$ signaling. Exp Cell Res 348: 1-9, 2016.

20. Rajabi H, Alam M, Takahashi H, Kharbanda A, Guha M, Ahmad R and Kufe D: MUC1-C oncoprotein activates the ZEB1/ miR-200c regulatory loop and epithelial-mesenchymal transition. Oncogene 33: 1680-1689, 2014.

21. Roy LD, Sahraei M, Subramani DB, Besmer D, Nath S, Tinder TL, Bajaj E, Shanmugam K, Lee YY, Hwang SI, et al: MUC1 enhances invasiveness of pancreatic cancer cells by inducing epithelial to mesenchymal transition. Oncogene 30: 1449-1459, 2011. 
22. Chen $\mathrm{CH}$, Chuang SM, Yang MF, Liao JW, Yu SL and Chen JJ: A novel function of $\mathrm{YWHAZ} / \beta$-catenin axis in promoting epithelial-mesenchymal transition and lung cancer metastasis. Mol Cancer Res 10: 1319-1331, 2012.

23. Shedden K, Taylor JM, Enkemann SA, Tsao MS, Yeatman TJ, Gerald WL, Eschrich S, Jurisica I, Giordano TJ, Misek DE, et al Director's Challenge Consortium for the Molecular Classification of Lung Adenocarcinoma: Gene expression-based survival prediction in lung adenocarcinoma: A multi-site, blinded validation study. Nat Med 14: 822-827, 2008

24. Booth CM and Shepherd FA: Adjuvant chemotherapy for resected non-small cell lung cancer. J Thorac Oncol 1: 180-187, 2006.
25. Gandara DR, Wakelee H, Calhoun R and Jablons D: Adjuvant chemotherapy of stage I non-small cell lung cancer in North America. J Thorac Oncol 2 (Suppl 3): S125-S127, 2007.

26. Situ D, Wang J, Ma Y, Zhu Z, Hu Y, Long H and Rong T: Expression and prognostic relevance of MUC1 in stage IB non-small cell lung cancer. Med Oncol 28 (Suppl 1): S596-S604, 2011.

27. Kufe DW: Mucins in cancer: Function, prognosis and therapy. Nat Rev Cancer 9: 874-885, 2009. 